\title{
InVestigating the Value of DJ Performance FOR CONTEMPORARY MUSIC Education AND Sensorimotor SynChronisation (SMS) Abilities
}

\author{
$\bullet$ Feature Article $\longrightarrow$ \\ $\begin{array}{lll}\text { Douglas MacCutcheon } & \text { Alinka E. Greasley } & \text { Mark T. Elliott } \\ \text { University of Leeds (UK) } & \text { University of Leeds (UK) } & \text { University of Warwick (UK) }\end{array}$
}

ABSTRACT

\begin{abstract}
Two studies were conducted to establish a more complete picture of the skills that might be accessed through learning to DJ and the potential value of those skills for music education. The first employed open-ended methods to explore perspectives on the value of DJing for music education. The second employed experimental methods to compare the ability of DJs to synchronise movement to auditory metronomes. Twenty-one participants (seven professionally trained musicians, seven informally trained DJs, seven non-musicians) took part in both studies. Qualitative data suggested that all participant groups felt DJs learn valuable musical skills such as rhythm perception, instrumental skills, knowledge of musical structure, performance skills, and a majority agreed that DJing had equal relevance with other musical forms e.g. classical music. Quantitative data showed that informally trained DJs produced more regular timing intervals under baseline and distracting conditions than the other experimental groups. The implications of the findings for the inclusion of DJing into formal music curricula are discussed.
\end{abstract}

KEYWORDS: DJ performance; turntablism; music education; sensorimotor synchronization; distraction

Douglas MacCutcheon is a Marie Curie Early Stage Researcher (ESR) and PhD candidate in Environmental Psychology at the Department of Building, Energy and Environmental Engineering, University of Gävle $(\mathrm{HiG})$. He has a background is in musicology and music psychology. Current research investigates how the classroom environment (acoustics, signal-to-noise ratios) affects language and memory in bilingual children and children with mild to moderate hearing loss and forms part of the ongoing Marie Curie project, iCARE (ImprovingChildren'sAuditoryRehabilitation, $<\underline{\text { https://www.icareitn.eu }}>$ ). $<$ Douglas.MacCutheon@hig.se $>$

Alinka Greasley (PhD) is Associate Professor of Music Psychology, School of Music, University of Leeds where she teaches music psychology at all levels, and leads the MA Applied Psychology of Music programme. Her research lies mainly within the field of social psychology of music, and her interests mainly focus on people's experiences with and uses of music in everyday life, including musical preferences, music listening behaviour, electronic dance music culture and DJ performance practice. <

Mark Elliott $(\mathrm{PhD})$ is Assistant Professor at the Institute of Digital Healthcare, University of Warwick. His research interests focus on human movement coordination. In particular, Mark's work has investigated how humans use the sensory information available to them to time and synchronise their movements.<M.T.Elliott@warwick.ac.uk>

\footnotetext{
Dancecult: Journal of Electronic Dance Music Culture 8(1): 46-72

ISSN 1947-5403 @2016 Dancecult http://dj.dancecult.net http://dx.doi.org/10.12801/1947-5403.2016.08.01.03 


\section{INTRODUCTION}

Traditional approaches to music education have been irrevocably transformed by the emergence of Information and Communication Technology (ICT) in schools as increased use of music technology to develop critical computer skills has introduced contexts to the music classroom that have not traditionally been associated with music and learning (Hallam and Rogers 2010; Himonides and Purves 2010). The varieties of music technology that children now have access to, and engage with, outside of the music classroom encompasses too broad a range of instruments, multimedia interfaces, software applications and creative workstations for an exhaustive list to be given here. The important issue is that the old methods of music education, such as those associated with traditional formal training, are not necessarily applicable to the educational contexts in which musical skills are now developed (Himonides and Purves 2010), and arguably are equally as inapplicable to new music styles and new instruments that have emerged out of contemporary technology and musical practices.

Music pedagogues in the UK and elsewhere have are becoming increasingly interested in the capacity for informal learning practices to inspire personal and creative growth in the music classroom, which has led to a growing inclusion of informal learning methods in music education in an attempt to ground music education in a more "learner-centred way" and combat the flagging interest in formal music training in schools (Price and D'Amore 2007; Green 2009; McQueen and Hallam 2010; Cain 2013). These changes in teaching and learning approaches have been well documented. For example, in her influential research, Lucy Green (2009) describes her experiences introducing informal learning practices of popular musicians into classroom environments in seven schools in Northern England, and in doing so, emphasises the teacher's role in guiding behaviour and tasks, and observing with minimal intervention or participation (Cain 2013). One UK initiative, Musical Futures, sees teachers as integral in exchanging knowledge about and embedding informal teaching and learning practices into music classrooms. The movement reflects increasing awareness of how learning has been radically altered by the influx of technology, both in and outside the music classroom, and a desire to adapt teaching styles accordingly (Thomas and Brown 2011). In 2010, McQueen and Hallam reported that Musical Futures included twenty eight schools in the United Kingdom and over seven hundred secondaryschool teachers (McQueen and Hallam, 2010); the initiative has subsequently expanded to Australia and Canada and is growing daily in scope and influence. Comprehensive reports of their research (including quantitative surveys and longitudinal studies) are available on the Musical Futures website. Results show that informal learning practices are adaptable to more formal educational contexts, and that the underlying processes are equally capable of producing musical outcomes as more traditional methods.

Although Green and the Musical Futures initiative focused on traditional instruments, this research argues that informal musical practices associated specifically with ICTs such as DJing/turntablism (terms used interchangeably throughout this article), may have the 
potential to develop critical creative and musical skills in learners as effectively as more traditional methods and instruments. DJing is a fairly diverse area covering a range of behaviours that have not necessarily been clearly delineated. However, there seems to be consensus in popular DJ tutor books as to how to learn to DJ (Broughton and Brewster 2002; Webber 2007; Steventon 2010; Vorobyev, Coomes and Murphy 2012). Smith’s (2013) investigation of "collective creation" among professional hip-hop turntable teams points towards an agreed consensus as to methods employed during DJ performance, suggesting a homogeny in approach among DJs that makes collaboration and learning possible. Due to this consistency of approach, DJ performance may perform a valuable function in music education classrooms in the future, particularly those that utilise collaborative and informal teaching and learning practices (Crow 2006).

While there is a growing body of literature on DJ culture (Bell 2009; Hansen 2010; Montano 2010, 2013; Attias, Gavanas and Rietveld 2013), DJ performance as a musical practice has been somewhat overlooked in music education and psychology literature in the UK despite its prevalence. The progressive increase in DJing outside the music classroom due to affordable technological advances has aided the growth of this informal music practice. This research posits that DJing might be utilised in the music classroom to convey essential music principles to learners who would otherwise feel alienated or restricted by more traditional instruments, may not come from backgrounds within which they are exposed to this subcultural musical practice, do not have access to the equipment due to socio-economic factors, or may be excluded from musical activities involving traditional instruments due to physical restrictions or disabilities.

\section{DJING IN CONTEMPORARY MUSIC RESEARCH AND EDUCATION}

DJs in their contemporary state evolved from two wellsprings. One source can be traced to the disk jockey radio announcers, influential cultural icons that selected and cued the records on music programs (e.g. Dick Clark and Alan Freed), while the second origin of DJing is traced back to the first experimental music written for the gramophone by the composer Hindemith in 1930 (his grammophonplatten-eigene Stücke) in which pitch/speed augmentation and sampling (layering two or more pre-recorded sounds simultaneously) were used in a recorded musical work for the first time (Katz 2012). When mixing two or more songs/samples simultaneously, contemporary DJs make use of techniques such as cuing, beatmatching, blending, phrase matching and rhythm matching (Broughton and Brewster 2002), techniques that are described in more detail in due course. In addition, some DJs use a set of two or more turntables as a musical instrument by scratching, negotiating and deconstructing the sampled music and "resisting the intended purpose of the turntable as an autonomous playback device" (Vandemast-Bell 2013: 243), thereby turning DJing into a virtuoso performance art which is referred to as turntablism (Chapman 2012). DJing is generally associated with hip-hop and electronic dance music (EDM: techno, house, trance, garage, drum n' bass, to name but a few) but the origins of turntablism as an art 
form are to be found in hip-hop (Poschardt 2002; Butler 2006). In hip-hop performances, the DJ mixes samples and breakbeats (percussive music samples) over which a vocalist raps, and performs occasional scratch solos on the turntables. Breakbeats have been used since the 1970s when DJs began to notice the effect of overtly percussive sections of music on disco dancers (Smith 2000). Studies in the psychology of music are beginning to investigate why percussive music like breakbeats elicit such strong responses in dancers (Van Dyck et al. 2013). DJing has also found its way into popular music genres in a variety of ways; for example, famous bands such as Portishead, Incubus, Linkin Park and Slipknot all feature live DJs as permanent band members.

An overview of the small quantity of current academic literature on DJing indicates that the there is still much about this musical practice to investigate within discipline of music psychology. A highly theoretical analysis of the semiotic content of DJ performance (specifically reggae and club DJing) has been provided by Bakker and Bakker (2006) which adopts a semiotic framework to interpret the meaning of DJing, but makes claims about what DJs do without any empirical grounding such as questionnaires and interviews. A recent addition to the academic literature on DJs is an article by Vandemast-Bell (2009, 2013), a DJ and academic. Bell used himself as the subject and discusses the manner in which live DJ performance involves tactile manipulation of sound through physical means, as well as "realtime composition". He is responding to the prevalent conception that a DJ is just someone that plays other people's music. Although his work provides an important context for this research, it is limited because it is based on the personal experience of an expert as opposed to objective data or scientific observations (Jabusch 2006). Further context for this research is provided by a study by Greasley and Prior (2013) which investigated the understanding and use of the notion of musical shaping among professional DJs. Results showed that DJs use the idea of shaping to modify a track while it is playing (e.g. equalisation); to control the transition between tracks (e.g. matching structural sections); and in relation to the overall trajectory of a set (e.g. choices of tunes, which order). There was also evidence that the DJs understood musical shaping multi-modally, through gesture and visual representation as well as sound. Of direct relevance to this study, Snell (2011) discussed the skills developed in turntablism courses at two higher education music departments-Berklee College of Music and the Royal Conservatory of Music in Toronto-in an attempt to illustrate the benefits for music educators and students. She established that skills developed were predominantly "beat matching, pitch awareness, improvisation, and sensitivity to musical styles" (Snell 2011: 176), and also argued that turntablists develop transferable skills. Studies such as these have provided important insights into what may be developed through DJing as an informally learned musical practice, yet more empirical research is needed. Tackling this need, Butler and Trainor (2015) quantitatively established DJs ability to detect rhythmic deviations in musical patterns were the same as trained percussionists, and also established whether or not a one-week intervention consisting of DJing lessons improved rhythmic pattern deviation detection - the results were non-significant. 
The purpose of the two music- studies reported in this article is to explore the relevance of DJing for music education, and to empirically establish a more complete picture of the skills that might be accessed through learning to DJ. In order to legitimise its use as an educational tool, this study aims to investigate basic motor skills developed by DJing (sensorimotor synchronization), and whether or not learning to DJ can be used to convey essential underlying musical principles.

\section{Study 1: Perspectives on the Value of DJ performance for Music Education}

The first study we devised sought to pinpoint contemporary perspectives on the value of DJing for music education, and equally sized groups of formally trained classical performers, informally trained DJs and non-musicians were recruited. A musical training questionnaire was used to determine the respective extents of our participants' musical training so as to make sure that our participants properly matched the respective categories of our study. All of the participants completed the perspectives on DJing questionnaire, and the DJs also took part in semi-structured interviews. The questions included perceptions of whether DJs can be classified as musicians, what musical skills they learn, and whether DJing should play a greater role in contemporary music education. The sample consisted of seven professional string players (age range 18-60, mean age $=26.56, S D=13.78$ ), seven non-musicians (age range 20-37, mean age $=25.43, \mathrm{SD}=4.89$ ) and seven professional DJs (age range $25-43$, mean age $=35.71, \mathrm{SD}=5.33$ ). The $\mathrm{DJs}$ performed in a wide variety of EDM styles (including electro, breakbeat, techno, house, hip-hop and drum ' $n$ ' bass, scratching) and with the exception of one participant who had attended a DJ academy for a short period of time, the DJs were all self-taught and randomly selected through opportunity sampling. Data were analysed using thematic analysis. Ethical clearance was gained from the Ethics Committees at the two institutions where the study was run, and in accordance with our ethics agreement, participants are referred to by their category (classical musician, DJ and non-musician) in the analysis in order to maintain anonymity, unless they explicitly asked to be named.

\section{PeRCeIVEd Musicianship of DJS}

The first line of enquiry concerned whether participants perceived DJs to be musicians. Twenty out of twenty one participants felt that DJs can be considered as musicians. Reasons given for categorising DJs as musicians grouped into four themes, namely, manipulation of mood and emotion, musical knowledge, technical knowledge and creativity. Some participants expounded on the musical knowledge employed by DJs, including rhythmic, melodic and structural elements as being essential to DJ practices. One classical string player felt that training was an additional necessity when defining a musician, while nonmusicians similarly thought that skill was essential. In a different but related question, one classical musician stated that DJs have to "master technology in a way similar to learning an instrument". One of the professional DJs made a direct comparison between DJing and piano playing during his interview: 
When you watch DJ Craze it's like a musician playing an instrument, it's like he's playing the piano, he's got all these beats set up, plus he's using the turntables and scratching the beat, and he was scratching with one hand whilst he was playing the beat with the other hand, like playing the piano, hands doing separate things.

Speaking of the creative processes behind DJing that substantiate the view of DJs as musicians, another DJ argued that "creating new music/a new song by combining pre-existing songs or using electronic sounds is no different to music created by the combination of different physical instruments or voices". In other words, a conventional musician plays patterns of musical tones with their instrument much the same way as DJs play patterns of samples (sections of recorded music) using their instrument, and as such, DJs situate themselves at a point somewhere between composer and musician through the act of (re)arranging presampled music in real time, often through improvisation. One DJ supported the general view, arguing "if they [DJs] aren't musicians, who play music, what could you classify them as?”. Only one (non-musician) participant openly disagreed that DJs are musicians, stating rather dismissively that "there is some skill involved and required, but that's the same for driving a car". Overall, participants' accounts suggest that DJs are perceived of as musicians if they physically mediate the sounds that are heard through various techniques (mixing and scratching for example).

\section{DJ Performance in Music education}

We asked the three groups of participants three questions relating to the value of DJing for music education: 1) whether they thought that DJs learn valuable musical skills through what they do, 2) whether DJing has cultural relevance on a par with classical music tradition, and 3) whether DJing lessons should be offered alongside traditional music lessons as part of a formal music education. In response to the question one, all participants unanimously agreed that DJs develop musical skills. Table 1 shows the skills mentioned in thematic categories. Only the category of mixing is DJ-specific; all other categories apply generally to music education.

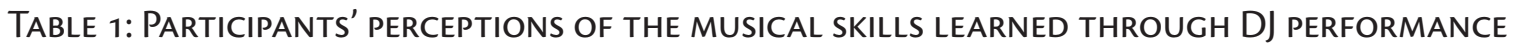

\begin{tabular}{|l|l|}
\hline Musical skill & Times mentioned \\
\hline Rhythmic perception & 7 \\
\hline Harmonic perception & 5 \\
\hline Musical structure & 4 \\
\hline Music technology & 4 \\
\hline Music performance & 4 \\
\hline Stylistic knowledge & 4 \\
\hline Instrumental skills & 3 \\
\hline Mixing & 3 \\
\hline Music production & 3 \\
\hline
\end{tabular}


Across all participant groups, rhythm perception was the most noted category of musical skill learned by DJing, perhaps as a result of the beat-based nature of the music and the fundamental necessity of beatmatching. This result was in line with the propositions of this research as a whole and substantiates the quantitative investigation of rhythmic perception described in Study 2. Mixing requires that harmonies as well as beats match, which might explain why skills relating to the category of harmonic perception were mentioned second-most by participants. Skills relating to musical structure, technology, performance and stylistic knowledge were all mentioned an equal number of times. In addition to a wide genre-based knowledge of available repertoire, DJs are required to understand the structure of bars, phrases and overall pieces in order to produce their mixes, while their knowledge of music technology and musical performance are obvious components of their art. Instrumental skills, mixing and music production were all mentioned three times. One professional DJ and tutor, Dan de Lissandri (henceforth Dan), felt the following skills were learned through DJing:

The most basic skill all DJs who mix have to learn is how to count to a pulse, how to recognise the first beat of a bar and phrase, and how to match the tempo of a song to the tempo of another song. Advanced musical skills occur when a DJ recognises the harmonic, rhythmic or textural effect of mixing two sounds together. Scratch DJs learn at least the equivalent amount of musical skills as percussion players as both use tactile methods to add musical elements to compositions. True turntablists use mixing, beat matching and scratching (i.e. all the elements of DJing) to produce entirely new compositions. These turntablist compositions utilise melodies, beats and basslines from other recordings but rework them into brand new pieces which are clearly separate from the original. There can be no doubt that these DJs have learnt valuable musical skills to do so (de Lissandri 2014).

Another professional DJ and tutor Jim Reiss (henceforth Jim) wrote:

Some may think it is just about pressing a button or to look good but there is a lot in involved in DJing; counting beats and bars, understanding structure and timing, learning about the key of a piece of music, musicianship, performance, music technology and creativity (Reiss 2014).

In the second question, participants were asked whether DJing had a cultural relevance on a par with classical music tradition. Out of the twenty one participants, nine stated that DJing had greater contemporary relevance than classical music, eight stated that DJing had equal relevance to classical music, and four claimed that DJing was not as relevant as classical music. Results were surprising; all classical musician participants claimed that DJing was equally as, or more culturally relevant than classical music, whilst those claiming DJing is less culturally relevant entirely consisted of non-musicians and DJs. It was expected that each group would defend their position (classical music for classical musicians, DJing for DJs) and non-musicians' opinions would gravitate towards their stylistic preferences (which were also 
noted as part of the study by correlating their responses with a list of each participant's top five favourite songs/albums), but results suggested that this was not the case.

\section{Should DJ lessons be Part of Formal Music Curriculum?}

The final question was intended to establish if people felt that DJing lessons should be offered alongside traditional music lessons as part of a formal music education. Sixty two percent of participants stated that DJing lessons should be offered, fourteen percent claimed that they should be optional, fourteen percent claimed that they did not know, and ten percent stated that DJing lessons should not be offered alongside traditional music lessons (both of whom were non-musicians). A non-musician participant stated that DJing is "more of a hobby and if you get good you could go professional but I don't think it's really an educational thing". This contrasts sharply with one DJ who argued that learning to DJ is as valid as studying the violin or piano because young people get to work creatively with the music they really enjoy, rather than having to learn predetermined repertoire, usually chosen by a teacher, and perform in a limited number of musical styles (e.g. Baroque, Classical, Romantic and Modern). Jim argued that the two supposedly disparate learning approaches could be mutually beneficial:

Most young people today gain their first access to music through pop culture and DJing is a large part of that. If we insist that all young people who show an interest in music need to learn a traditional instrument first, then we will fail to encourage musicianship in the majority. If we do offer DJ education we help naturally musical young people discover music in a manner which they feel comfortable with. This in turn helps us signpost them onto more traditional instruments if they so wish (Reiss 2014).

Another DJ noted the functional value of DJing skills by mentioning that "DJing has a place in the music industry; therefore, you are equipping people with skills to work".

The two DJ tutors Jim and Dan were DJing instructors at a DJ school and were active in local schools where they were using the teaching of DJing skills for various educational purposes that were partly funded by local council for certain projects. Their opinions on the value of DJing in music education were highly developed and their answers provided substantial arguments for inclusion of DJ performance in school music curriculums. Discussions centred on the motivational aspects of DJing for young people, including children with Special Educational Needs (SEN); devising a rubric for teaching DJing in a consistent way, which differs according to the style being taught: e.g. the 'choppy' style of hip-hop mixing compared with 'seamless' mixing style of progressive house, and the inclusion of DJ performance into mainstream education. Their first focus when teaching was to get the young people thinking about the preferred music they personally would want to hear in a mix. The tutors were very keen to impress on the young people that they should choose music that they enjoy. The tutors then teach the basics of mixing, acknowledging that this differs according to different musical styles, as emphasised by Dan: 
We start off by teaching about bringing the record in on the first beat, then we explain the structure of the music, because we try and tie it into their music lesson as well, getting them to think about the intro, when you listen to a piece of music, you get an introduction, you might get a hook, a riff, a break, verse, chorus, another break, so getting them to understand that, and we've devised a diagram [see Figure 1] to show the layering of tunes ...I want to get some lego actually, to try and explain it better, a few blocks of lego would be one track, and then you overlap it with another track, how to work out where to bring the next track in, analysing that there's a minute introduction, and so on, but at the same time, it's entirely up to you how you mix it, it's your creativity, your originality, we're just explaining how we do it, how other DJs do it, or explaining how the music has been written, but there's no rules around that...that [points to diagram] approach works with house music, and drum and bass, but with hip-hop and $\mathrm{RnB}$, or popular or rock music, a different mixing style is needed, maybe just fading it, or doing an effect just before dropping the next tune in (de Lissandri 2014).

The tutors noted that tune selection was particularly important when working with young people with physical disabilities, many of whom did not have the motoric skills needed to manipulate the speed of the platters on the decks. Overall, the tutors felt that around ninety five percent of the young people they worked with were able to gain at least basic skills, and they talked about how motivating and confidence building DJ performance could be, particularly for children from disadvantaged backgrounds, and SEN children.

As regards the relevance of DJing for the traditional music curriculum, they argued that they "tick lots of curriculum tick boxes, such as understanding musical structure, dynamics, texture, and creative processes" (Reiss 2014) and that interest in DJ lessons was growing all the time. They were applying for further funding to allow them to work in a greater range of schools, continue the outreach work they had started, and raise awareness among secondary school teachers. When asked how realistic it is that DJing could start to form part of the curriculum, Dan responded:

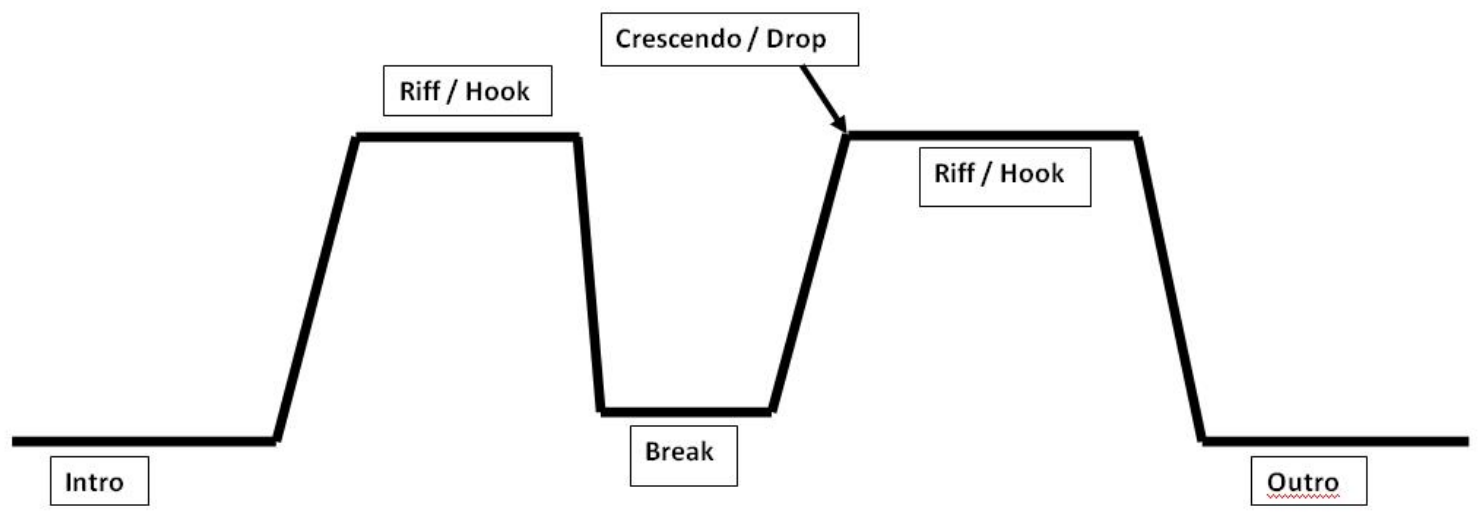

Figure 1. Dance Music Song Structure (Simplified for teaching purposes) 
I really do think it will. From us speaking to secondary school music teachers, and Heads of music, because we've recently taught at ten schools around the city, speaking to most of them, a lot of young people are interested in DJing as their performance for GCSE, but the music teachers don't understand it enough, the examining board don't understand it enough, so it's a bit of a difficult situation for them (de Lissandri 2014).

It became apparent both from the tutors' accounts and the responses from all participant groups about the relevance of DJing that there is a need to establish criteria for the examination of DJ performances to enable young people to pursue DJing at school.

The DJ tutors reported that future work included a conference linked to a higher education music college within the city in which they were running workshops for secondary school teachers to teach the basics of DJing and the range of DJ equipment that is currently available. At a six month follow-up, we asked Jim and Dan how the workshops-which encompassed how to plan a mix, demonstration of mixing and scratching, 'have a go' and Q\&A sessions-had been received by the teachers. At the start and end of each workshop, they asked teachers to list disadvantages and advantages of DJ performance. Teachers were focused on disadvantages at the start, listing only a few advantages, including cost of equipment, lack of teacher understanding, and problems with assessment, but were much more focused on advantages at the end, such as improved aural and timing skills, and musical creativity (see Table 2). After the sessions, the tutors had immediate bookings from schools to deliver workshops to their pupils.

TABLE 2: TEACHERS' PERCEPTIONS OF THE ADVANTAGES AND DISADVANTAGES OF DJING AT GCSE LEVEL

'Pros' before session
"Inclusive"
"Accessibility. Cultural relevance".
"Exciting. Relevant to their interests. Not stuffy".
"Cool and instant. Leading to depth as appropriate".
"Getting kids interested in engaging with music who might not have otherwise".

\section{'Cons' before session}

"Large amount of songs and software will be trouble for crappy school networks". "Many music teachers don't understand DJing".

"Cost? £”.

"How to assess a performance? Too 'informal' for an exam".

"Not enough educational material to inform teaching / learning". 


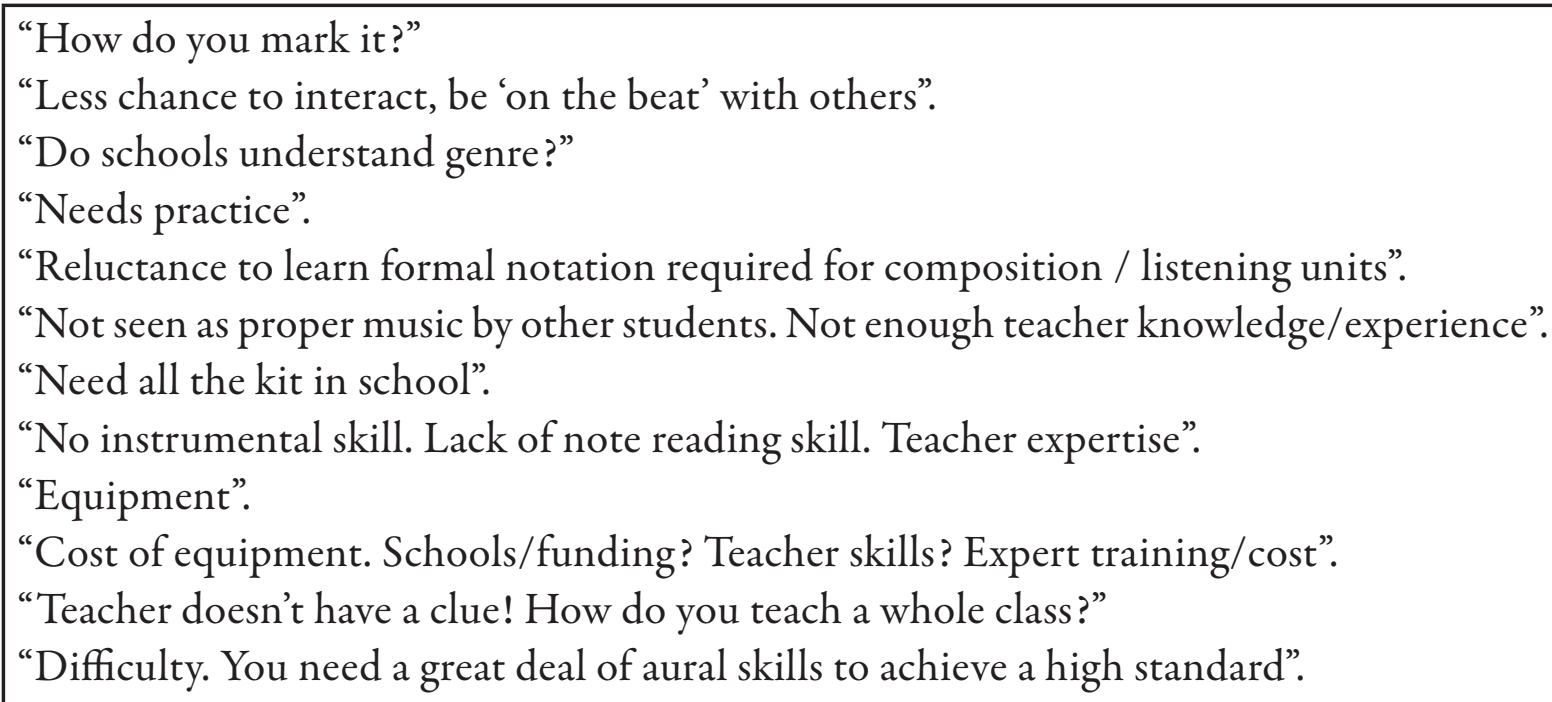

\section{'Pros' after session}

"Understanding of tempo and tempo relationships (cross curricular links with numeracy)". "Permits access to technologically / scientifically minded kids".

"Engages challenging students. Own musical interests addressed. Engaging with music more accessible”.

"Improved aural skills. Develops ICT skills. Accessible music. Engaging. Cool".

"Real musical creativity possible".

"Timing. In-tune. Engaging."

"Ability to access music course through something they enjoy".

"DJing can be a form of an arrangement."

"Engaging for kids - lots of DJs in mainstream at the moment".

"Genre accessible to some young people who are harder to reach with "conventional" instruments”.

"Engaged. New skills."

"Range of options for equipment. Lots of analysis and skills involved. Engaging and creative".

"Affordable - for the entry versions".

"Enthuses. Encourages practice and perseverance".

"Fun to do".

"Addictive! Inspiring. Challenging".

"Rhythmic manipulation and splicing of breaks to form a new piece. Restructuring preexisting materials to create a new form".

"Cost effective to beginners for first time equipment".

"Naturally engaging. Immediately musical". 


\section{Study 2: The Sensorimotor Synchronization (SMS) ability of DJS}

In our second study, we devised an experiment to explore how accurately DJs can time and coordinate their movements in comparison to formally trained classical musicians and non-musicians. The experimental paradigm used for studying this is often termed as sensorimotor synchronization (SMS), or the "coordination of rhythmic movement with an external rhythm" (Repp 2013: 403). This task is of particular importance because it is the fundamental cognitive ability that allows humans to engage in nearly all aspects of musical performance, and extends to all forms of synchronised movement across species (Toiviainen and Snyder 2003; Jäncke 2006; Repp and Su 2013). Sensorimotor synchronization (SMS) is often studied by measuring participants' finger tapping responses to an external auditory metronome, presented under a variety of conditions (Repp 2005; Repp and Su 2013).

There are many contexts in which this sort of behaviour may occur in ecologically valid contexts, but a rhythmical musical setting remains the most obvious and prevalent environment in which this cognitive-motor skill is applied, as it aids synchronization between players in collaborative musical activities (Repp 2005; Jäncke 2006;). SMS is also necessary for DJing, particularly when beatmatching, cuing and scratching.

Tapping along to an isochronous (regularly occurring) beat is a relatively easy task for most people. There is evidence that correcting and maintaining movements in time with an external isochronous auditory beat is automatic. The timing errors observed between the movement and the external rhythmic cue are referred to as asynchronies and the process human subjects apply to minimise their asynchronies are collectively referred to as error correction in the SMS literature (Repp and Su 2013), which includes phase correction and period correction (Repp and Moseley 2012; Repp and Su 2013). Phase correction consists of the automatic process of adjusting of the phase of subsequent taps relative to the phase of the stimulus onsets (Repp and Moseley 2012), and in this way, synchronization is established through continuous sensory feedback (Engbert et al. 2002). This is referred to as the phase correction response (PCR) (Repp 2003a). Period correction is a voluntary error correction action and consists of the subject comparing the perceived inter-onset interval (IOI) of the stimulus (i.e. the metronome clicks) with the period of their "inner timekeeper" or internal sense of the beat, and correcting the inter-response intervals (IRIs) (i.e. the distance between subsequent tapped responses) accordingly (Repp and Su 2013).

SMS studies have highlighted significant differences in timing accuracy between different populations and under a variety of different conditions that test the extent of this ability within and across modalities (Repp 1999, 2003b; Krampe et al. 2001; Aschersleben 2002; Krause et al. 2010a; Elliott et al. 2011; Fuji et al. 2011). Musicians have been shown to be highly advantaged in this task (Aschersleben 2002; Repp 2003b) and some musicians more than others (Krause et al. 2010a, 2010b; Fuji et al. 2011). In contrast, musically untrained people have been shown to be an appropriate control group for SMS studies because their asynchronies tend to be approximately $10 \mathrm{~ms}$ larger than amateur musicians (Aschersleben 1994), and even greater when compared with music students (Aschersleben 2002). In 
addition to being more accurate, musicians have been shown to be less variable in their tapping responses than non-musicians in a variety of studies (Madison 2000; Repp and Doggett 2007; Krause et al. 2010b; Repp 2010; Fuji et al. 2011). These differences between groups provoke questions as to how SMS ability relates to the motor system, cognitive processes such as attention and perception, and how is it developed within different learning contexts such as formal and informal training. An ecologically valid musical setting requires a more complex application of SMS ability (Wing, Endo, Bradbury and Vorberg 2014). In this context, continual adjustment of timing takes place in order for performers to convey the expressive intentions of the music and maintain interpersonal coordination with other performers through the cognitive processes of prediction and adaption (Konvalinka et al. 2010; Nowicki et al. 2013). A central tenet of the current research is that in an ecologically valid performance setting, SMS adaptability has to be maintained despite unexpected internal and external distractions that direct attention away from the target source. Previous studies show that a distractor-based experimental paradigm is the most applicable for testing this complex phenomenon in musicians, who are expected to perform more accurately under conditions of distraction than the control group of non-musicians (Repp 2003a; Repp 2005). Here, we will investigate the differences in SMS performance between formally trained (string players) and informally trained (DJs) musicians, using a distractor-based paradigm.

Professional string players were chosen to represent the formally trained group because of the degree to which they regularly entrain rhythmically with external sources (Repp 2005; Repp and Su 2013) in auditory and visual domains, without the direct use of some form of tapping with their dominant hand. For this reason, formally trained drummers and pianists were excluded from the participant group because tapping with their dominant hand is an intrinsic part of their art and provides a distinct motoric advantage in SMS tasks over other types of musicians (Krause et al. 2010a, 2010b; Fuji et al. 2011). Despite a lack of actual tapping with their dominant hand (which usually holds the bow), an ensemble musician in an orchestra synchronously entrains with the visual cues provided by a conductor as well as the auditory cues provided by the multitude of other instruments in their sonic environment through the perceptual/cognitive mechanism of feedback control (Jäncke 2006). In the execution of this process they must filter out the misleading sounds of those less capable of entraining due to differences in skill level, distractions and other factors, and focus on their own auditory stream as well as other auditory streams they perceive to be consistent with the conductor's timing-related gestures (Luck and Toiviainen 2006). DJs were chosen to represent informal musical practitioners because of the prevalence of DJing as a performance art and the degree of professionalism DJs attain without any formal musical training (Greasley and Prior 2013). As described above, the practical development of SMS occurs during musical performances when timing discrepancies brought about by distractions, differences in skill level between players and other factors are perceived and corrected by the performer. This ability to isolate and eliminate noticeable asynchronies successfully is especially relevant to the fundamental DJing technique of beatmatching, in which one beat must be strictly 
adhered to whilst matching the other (asynchronous) beat during a performance in order for two tracks to be appropriately mixed (i.e. played simultaneously). Although there are technically no other performers in a conventional DJ setup, the coordination and entrainment of two rhythmic sources in phase is the essential part of DJ performance that is thought to develop SMS ability, a central hypothesis of the quantitative study reported in this article. Furthermore, DJs are continually aiming to maintain synchrony in a performance by eliminating asynchrony between the beat of the music that is already playing, and the samples they overlay and "mix" into the sonic environment, which is at the heart of what DJs do: intertextual layering of different audio samples to produce new and interesting combinations and aesthetic relationships (Bradby 1993; Kistner 2006).

\section{HYPOTHESES}

Formally trained classical musicians achievegreater accuracy in sensorimotorsynchronization experiments, in terms of reduced mean asynchronies and lower asynchrony variability when compared to non-musicians (Repp and Doggett 2007; Fuji et al. 2011). We predicted that professional DJs would exhibit movement-timing performance that closely matched that of formally trained classical musicians, despite having been informally trained.

\section{METHOD}

We assessed movement timing performance of three groups of participants: non-musicians (NM), formally trained classical musicians (CM), and informally trained DJs (DJ). We used a sensorimotor synchronization paradigm to test the performance of each participant. In addition to a simple finger-tapping task to an auditory metronome, we also required participants to synchronise movements to a metronome in the presence of an additional "distractor" metronome. In this paradigm, participants are required to synchronise their taps as accurately as possible with an isochronous target sequence whilst an additional simultaneous isochronous metronome sequence, the "distractor", diverts attention away from the target sequence (Repp 2003a, 2005). In this experiment we used phase distractors. A phase distractor metronome has the same tempo as the target metronome, but is offset in phase, such that the beats of the distractor consistently occur slightly earlier or later than the beats of the target metronome. Distractor cues disrupt participants' movement responses by exerting the effect of phase attraction, causing participants to gravitate the timing of their movements towards the onset phase of the distractor metronome and away from the target phase onset, a phenomenon which participants have shown difficulty in resisting (Repp 2002). The phase distractor paradigm is a task that is very much related to the challenge faced by a DJ beat mixing two tracks: they must be able to switch attention between the independent beat-lines of the two tracks (Broughton and Brewster 2002). Hence, we predicted that participants in the DJ group would show superior performance in this task compared to the other two groups. 
The same twenty one participants who participated in the qualitative study took part in the experiment. Technical problems with the tapping sensor led to a substantial loss of data from one of the classical musicians. In addition, we found that during analysis another classical musician appeared to have struggled to synchronise with the metronome during the simple baseline tapping tests. Hence they were excluded from further analysis. To balance group sizes, we analysed the best five participants from the DJ and Non-Musician (NM) groups along with the five remaining classical musicians (CM). Selection performance was based on the lowest average asynchrony variability measured across all baseline trials.

\section{EQUIPMENT SETUP}

Participants sat a desk and were instructed to tap their index finger onto a force-sensitive resistor in time with an auditory metronome presented over Sennheiser HD-212 headphones. The experimental trials and metronome signals were controlled using the MatTAP toolbox (Elliott et al. 2009) in MATLAB (The Mathworks Inc., MA, USA). A National Instruments DAQ-USB 6229 data acquisition card was used to output the metronome cues outputs and also capture the tapping response inputs with a common 10k Samples/sec sampling clock. The presented metronome stimuli had a tone pitch of $440 \mathrm{~Hz}$, with each tone having $30 \mathrm{~ms}$ duration. In the Distractor conditions, an additional metronome was presented simultaneously, differing only in pitch $(700 \mathrm{~Hz})$ and was phase offset to the target metronome.

\section{EXPERIMENTAL TASK}

The experiment was split into two main tasks: a Baseline task and a Distractor task. During the Baseline task, participants synchronised finger taps to a single metronome. These baseline conditions were established in order to measure participants' asynchrony mean and variance under normal conditions, i.e. without distraction. For each trial, the metronome was presented at one of 6 different inter-onset intervals (IOI, the time between beat onsets, see fig. 2): $340 \mathrm{~ms}, 420 \mathrm{~ms}, 500 \mathrm{~ms}, 580 \mathrm{~ms}, 660 \mathrm{~ms}$ and $750 \mathrm{~ms}$. Each trial consisted of 30 metronome beats. Participants completed three trials per IOI condition.

In the Distractor task, the auditory stimuli consisted of a target metronome with an IOI of $500 \mathrm{~ms}$ in all trials. After an initial 5 beats of the target metronome, an additional distractor metronome was introduced (differentiated from the target metronome by a higher tone pitch). The distractor metronome had the same IOI as the target metronome, but the beats were offset in time relative to the target by: $\pm 160 \mathrm{~ms}, \pm 80 \mathrm{~ms}, \pm 40 \mathrm{~ms}, \pm 20 \mathrm{~ms}$ and $0 \mathrm{~ms}$. A positive phase offset meant the distractor metronome beats were presented slightly later than the target beats; a negative offset resulted in distractor beats presented earlier than those of the target metronome (See Figure 2). Each trial consisted of 30 target metronome beats ( 25 distractor metronome beats) and participants completed 3 trials per phase-offset condition. 


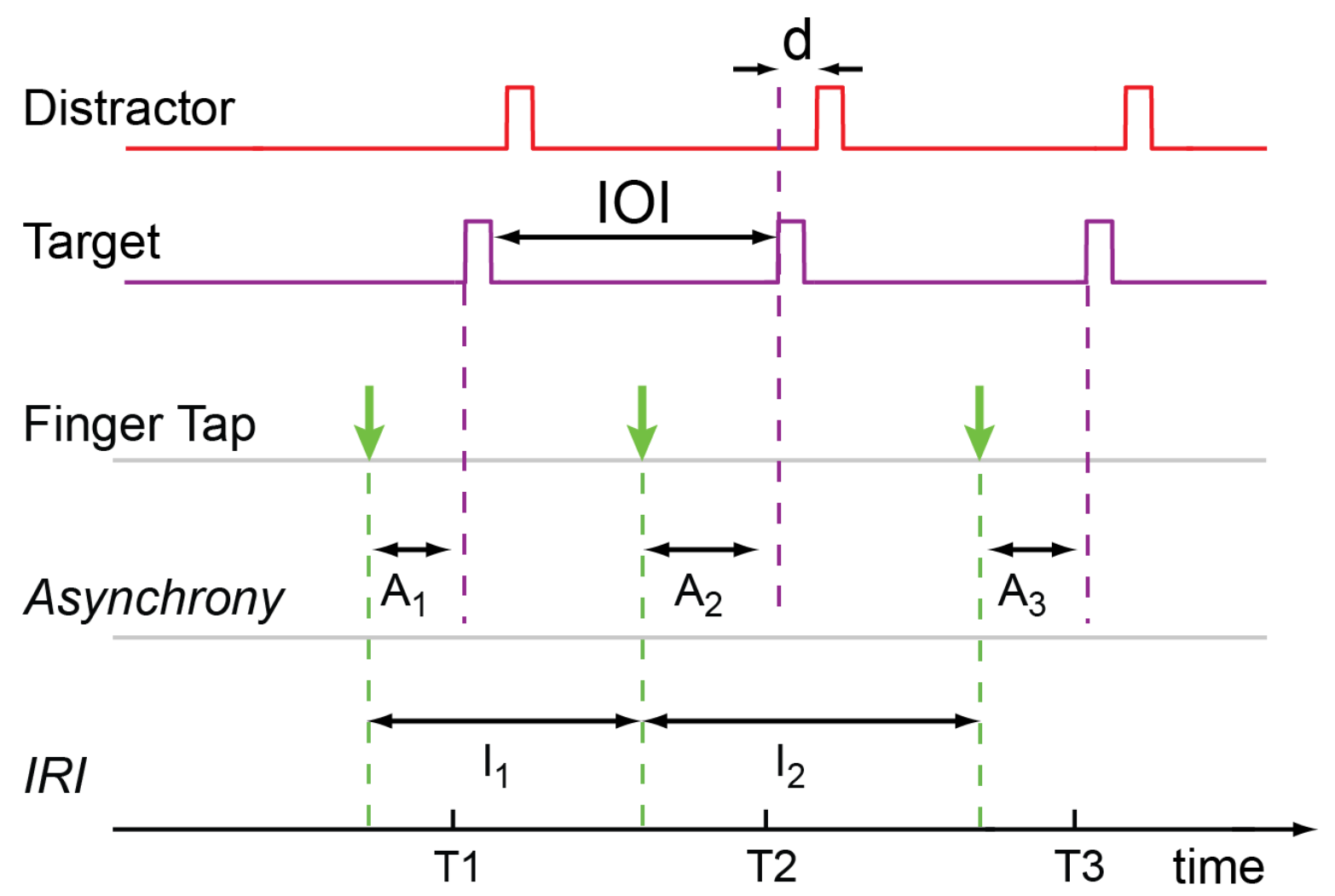

Figure 2. SCHeMATIC Representation of the EXPERIMENT. PARTICIPANTS WERE INSTRUCted to tAP their FINGER IN TIME TO A TARGET PURPLE METRONOME WITH AN INTER-ONSET INTERVAL (IOI) OF 500 MS. AN ADDITIONAL RED DISTRACTOR METRONOME WAS PRESENTED WHICH HAD THE SAME IOI AS THE TARGET BUT WAS OFFSET IN PHASE BY AN AMOUNT D, SUCH THAT EACH BEAT OCCURRED SLIGHTLY EARLIER OR LATER THAN THE TARGET BEATS. IN THE BASELINE CONDITION, ONLY THE TARGET METRONOME WAS PRESENTED AT A NUMBER OF DIFFERENT IOIS. FINGER TAP ONSET TIMES WERE RECORDED AND MEASURED RELATIVE TO THE TARGET METRONOME ONSETS TO CALCULATE ASYNCHRONY (A). EARLY FINGER TAPS (AS SHOWN) RESULTED IN NEGATIVE ASYNCHRONIES. INTER-RESPONSE INTERVALS (IRI) WERE CALCULATED AS THE TIME BETWEEN CONSECUTIVE FINGER TAP ONSETS.

Baseline and Distractor trials were interspersed and presented in a randomised order across the experiment. Participants were instructed that they would hear a single target metronome, followed by a second distractor metronome in some trials. Their task was to always tap along with the first metronome they heard and ignore the distractor metronome as best as they could. Participants completed two practice trials to familiarise with the task.

\section{ANALYSES}

We recorded the finger tap onset times and aligned them to the related target metronome beat onset times using the MatTAP toolbox (Elliott et al. 2009). From these measures we calculated the corresponding asynchronies and IRIs across a trial (see Figure 3). The mean and standard deviation of asynchronies and IRIs were subsequently calculated for each trial and averaged across trials within a condition for each participant. For the Distractor task, 
we further analysed the mean relative asynchrony to quantify how much participants were distracted away from the target metronome. That is, how much the asynchrony changed relative to the asynchrony observed in the $0 \mathrm{~ms}$ phase offset condition.

Baseline conditions were analysed independently from Distractor conditions. For the Baseline task, statistical analyses were based on a Group (DJ, CM, NM; between-subjects) $x \operatorname{IOI}(340,420,500,580,660$ and 750ms; within-subject) mixed-ANOVA design. For the Distractor task a similar mixed ANOVA design was used based on Group (DJ, CM, NM; between-subjects) x Phase Offset $( \pm 160 \mathrm{~ms}, \pm 80 \mathrm{~ms}, \pm 40 \mathrm{~ms}, \pm 20 \mathrm{~ms}$ and $0 \mathrm{~ms})$. Post-hoc analyses were adjusted for multiple comparisons using the Bonferroni method. GreenhouseGeisser adjustments were made for results that violated sphericity assumptions.

\section{RESULTS: BASELINE TASK}

As observed in most sensorimotor synchronization tasks (Repp 2005; Repp and Su 2013) we found all groups tapped slightly ahead of the metronome showing a negative mean asynchrony result regardless of the metronome IOI (fig. 3A). We found no significant difference in the mean asynchrony between groups $(\mathrm{F}(2,12)=.55, \mathrm{p}=.593)$, but did observe a slight change in the mean asynchrony in relation to the metronome $\mathrm{IOI}(\mathrm{F}(1.7,3.4)=4.76$, $\mathrm{p}=.024)$. In particular, we observed that the shortest IOI resulted in a more positive mean asynchrony ( $p=.030$, relative to $420 \mathrm{~ms}$ IOI; fig. 3B). Participants' tap intervals also corresponded very closely with the metronome with mean IRIs being within $1-2 \mathrm{~ms}$ of the metronome IOI. We found no significant difference in mean IRI between groups.

A

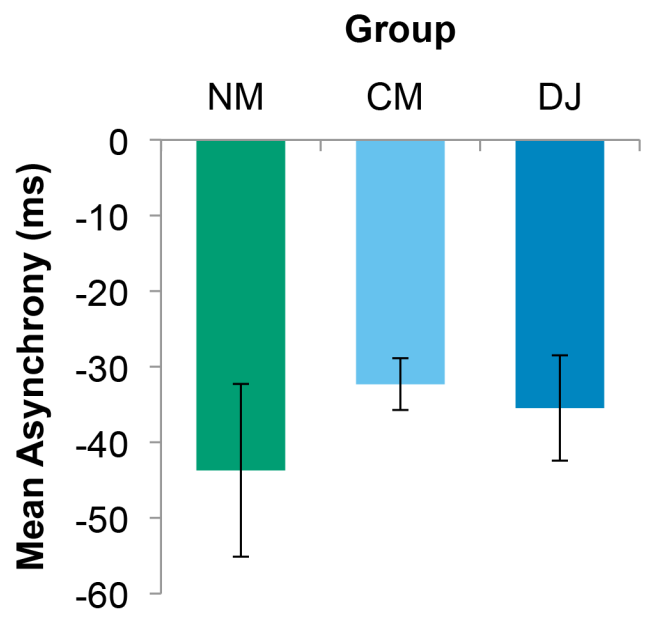

B

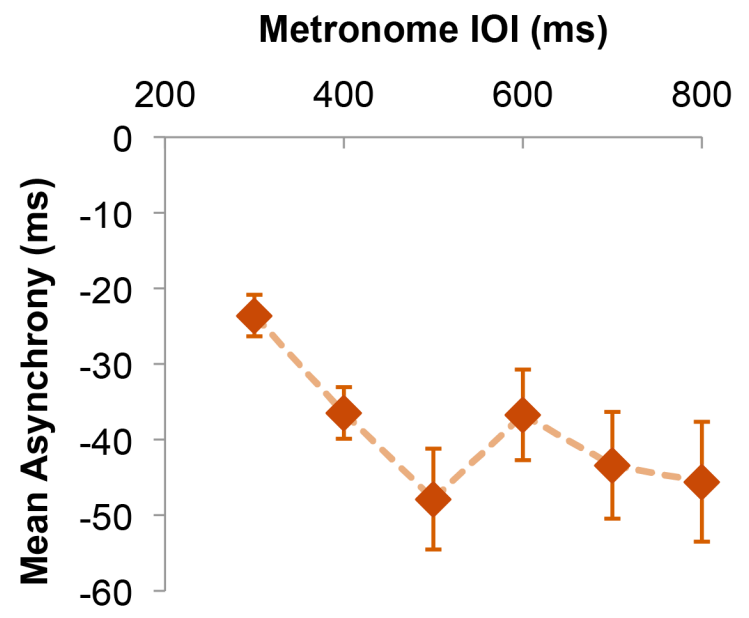

Figure 3. (A) MEAN ASYNCHRONY ACROSS BASELINE CONDITIONS BY GROUP. ERROR baRS INDICATE STANDARD ERROR (SE). (B) MEAN ASYNCHRONY BY METRONOME IOI IN BASELINE CONDITIONS, COLLAPSED ACROSS GROUPS. ERROR BARS INDICATE SE. 
Variability in production of the timing intervals did vary between groups $(F(2,12)=7.80$, $\mathrm{p}=.007)$. In particular, we found a significantly lower IRI standard deviation in DJs compared to both CM $(\mathrm{p}=.009)$ and NM $(\mathrm{p}=.029)$ indicating that participants in the DJ group produced more regular timing intervals (see Figure 4A). We observed a similar, non-significant trend for the asynchrony standard deviations across the three groups (see Figure 4B). As expected, slower metronome tempos (longer IOIs) resulted in higher IRI $(F(5,10)=23.29, p<.001$; see Figure $4 C)$ and asynchrony $(F(5,10)=20.61, p<.001$; see Figure 4D) variability regardless of group.

A

IRI

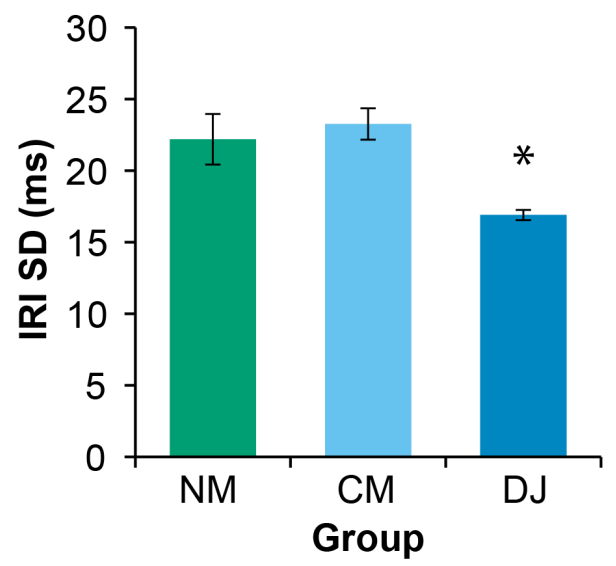

C

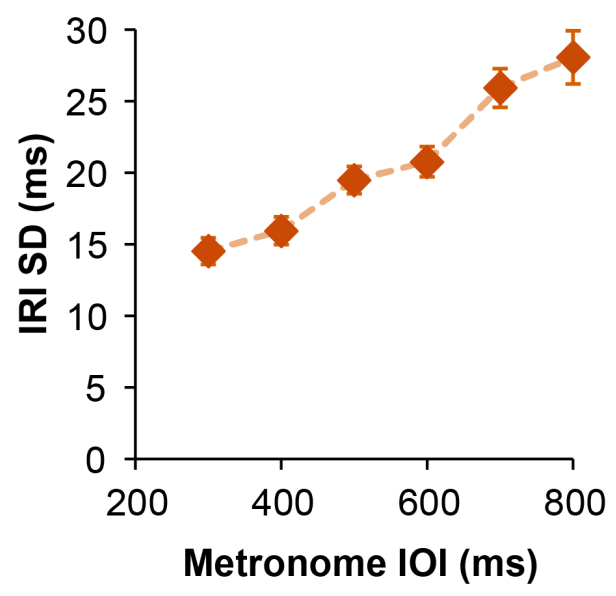

B

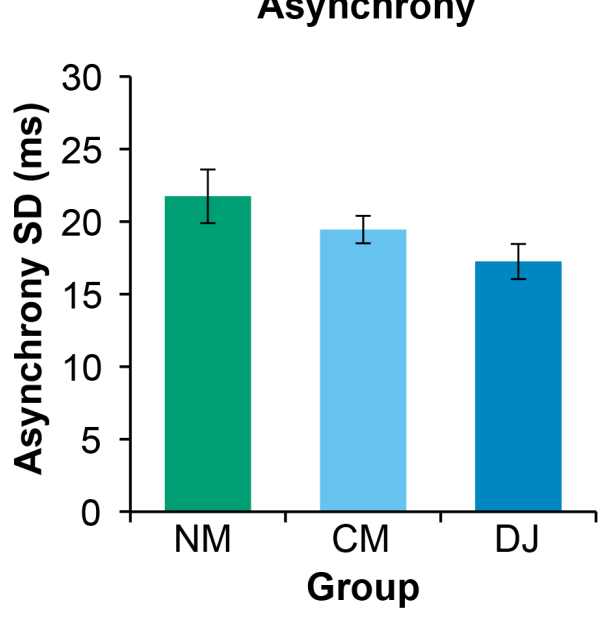

D

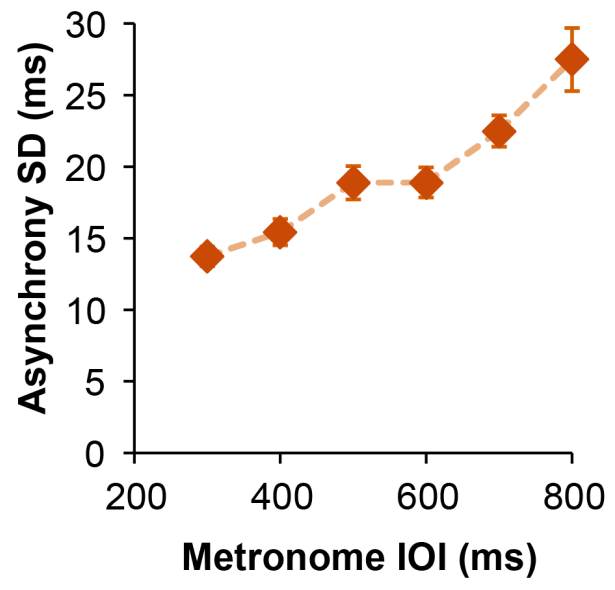

FIGURE 4. THE DJ GROUP HAD A SIGNIFICANTLY LOWER IRI VARIABILITY THAN THE NM AND CM GROUPS (A). A SIMILAR BUT NON-SIGNIFICANT TREND BETWEEN GROUPS WAS ALSO OBSERVED FOR ASYNCHRONY VARIABILITY (B). AS PREDICTED, BOTH IRI (C) AND ASYNCHRONY (D) VARIABILITY INCREASED WITH LARGER METRONOME INTERVALS. ERROR BARS INDICATE SE. ${ }^{*}=\mathbf{p}<.05$. 


\section{RESULTS: DISTRACTOR TASK}

To measure the effects of the distractor metronome on each group, we analysed the change in asynchrony as a function of the distractor phase offset. For each participant, we subtracted their mean asynchrony in the condition when the two metronomes were aligned (i.e. no distractor) from the mean asynchronies calculated in each of the other Distractor conditions. These relative asynchronies were then averaged across participants within each group and plotted against the phase offset of the distractor (see Figure 5). A least squares linear regression was applied to the resulting data. The gradient of the fitted line indicated how strongly the participants within each group were influenced by the distractor: a large positive gradient suggested the were strongly influenced, while a gradient close to zero indicated that participants were able to ignore the distractor metronome and continue to tap in time with the target metronome. We found that the NM group were particularly affected by the distractor metronome (gradient $=.26$, $\mathrm{R}^{2}=.78$ ), while $\mathrm{CM}$ were less affected (gradient $=.11, \mathrm{R}^{2}=.39$ ). The $\mathrm{DJ}$ group appeared to be unaffected by the distractors overall (gradient $=.00, \mathrm{R}^{2}=.00$ ). The low $\mathrm{R}^{2}$ for the DJ group suggests a poor fit. This is mainly due to a distractor effect occurring only at $-20 \mathrm{~ms}$ and $-40 \mathrm{~ms}$, suggesting distractors with a very small phase offset have a relatively strong effect, even in the DJ group.

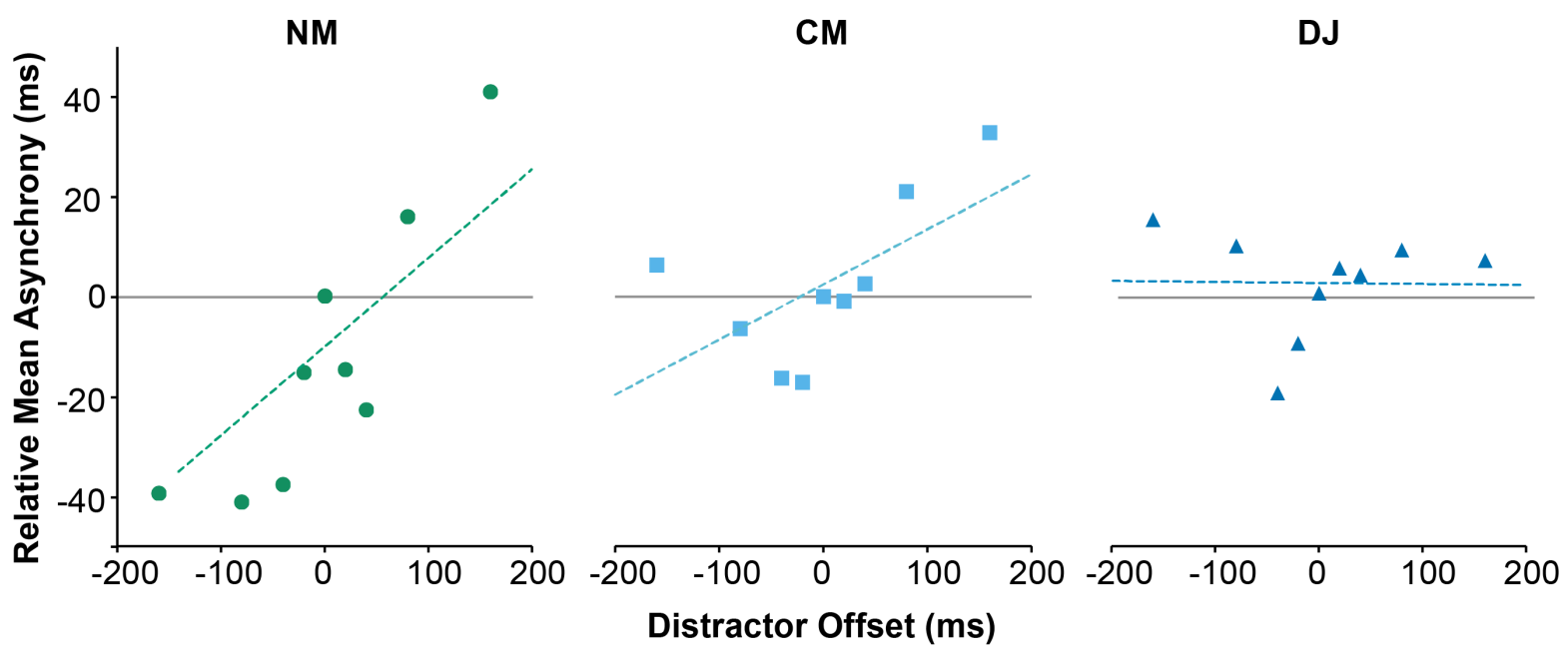

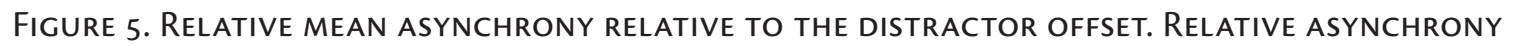
IS CALCULATED BY SUBTRACTING THE MEAN ASYNCHRONY AT ZERO DISTRACTOR OFFSET (I.E. NO DISTRACTION) FROM THE MEAN ASYNCHRONIES AT EACH OF THE OTHER OFFSETS. NEGATIVE NUMBERS INDICATE EVENTS OCCURRING EARLIER THAN THE TARGET METRONOME BEAT. A LEAST SQUARES LINE OF BEST FIT IS SHOWN ON EACH PLOT (DASHED LINE). THE STEEP GRADIENT OF THE NM GROUP INDICATES A STRONG EFFECT OF THE DISTRACTOR ON THE PARTICIPANTS' ASYNCHRONIES. IN CONTRAST, THE ALMOST FLAT GRADIENT IN THE DJ GROUP INDICATES THEY ARE UNAFFECTED BY THE DISTRACTOR CUE. 
Finally, we investigated how the distractor metronome contributed to the variability of the tapping asynchronies to the target cue. We found a significant interaction between phase offset of the distractor and Group $(\mathrm{F}(5.2,31.4)=2.81, \mathrm{p}=.031)$. Further investigation indicated that the DJ group achieved a lower asynchrony variability overall, compared to the NM group ( $\mathrm{p}=.015$; see Figure 6). In addition, we observed that for large positive distractor phase-offsets, the NM and CM groups both had a large increase in variability, while DJs were unaffected.
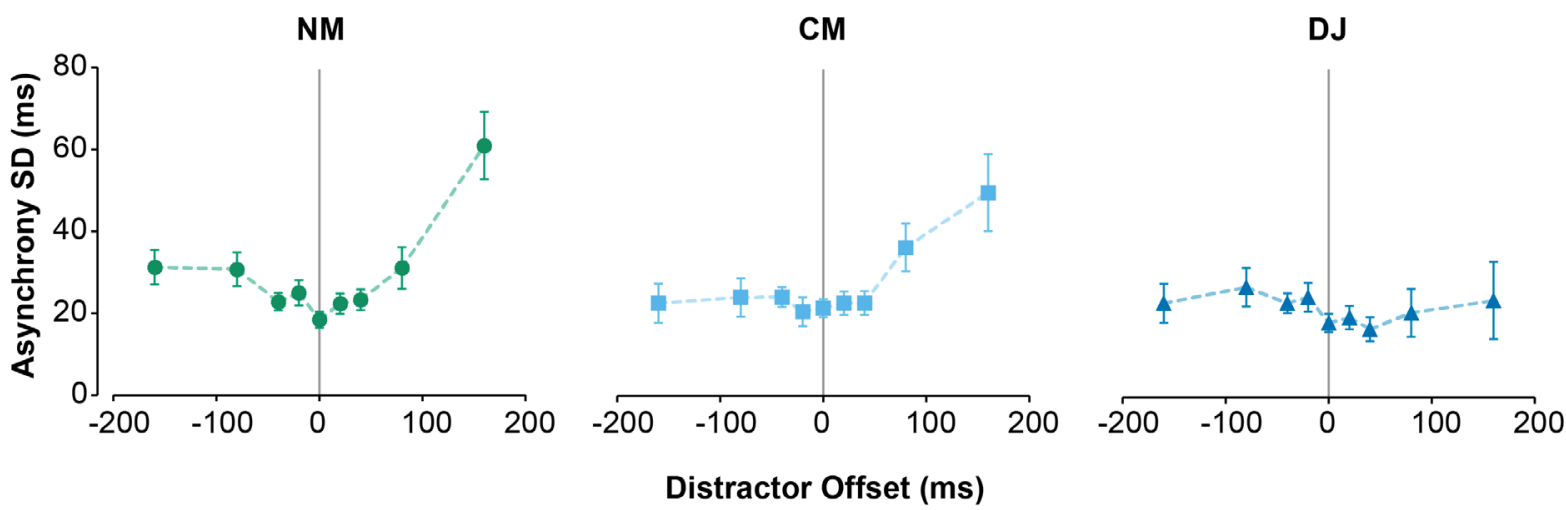

Figure 6. Asynchrony StANDARD DEVIATION RELATIVE to the Distractor offSET. Both NM AND CM GROUPS SHOW LARGE INCREASE IN VARIABILITY FOR THE +8OMS AND +16OMS DISTRACTOR OFFSETS. HOWEVER, THE DJ GROUP SHOW A CONSISTENT VARIABILITY REGARDLESS OF DISTRACTOR OFFSET.

\section{General Discussion}

DJing/turntablism and other informally learned arts and creative practices have already been used in a variety of contexts outside of formal education that substantiate their potential use-value in education and the wider community. For example, hip hop arts practices (which include turntablism, emceeing, break-dancing and graffiti) (Chang 2005) were utilised in a number of community-based projects in Canada which fostered "creative, thoughtful and artistic subjectivities" in children (Marsh 2012). Furthermore, Pasagiannis (2007) showed the therapeutic value of turntablism for "at risk" youths. Indeed, the value of including creative technologies and ICTs within Fine Arts and other disciplines has been well substantiated in the literature, and it is widely acknowledged that such practices are of substantial value in terms of promoting creative thinking (Wheeler et al. 2002), collaboration (Smith 2013), identity formation (Marsh 2012; Shiu 2007), both outside and within the classroom (Marsh 2006). However, this paper has focused on skills developed that are relevant with regards to conveying musical principles and instrumental techniques, as we believe that a case has already been strongly made with regards to extra-musical skills in the literature. 
In the quantitative study, all groups showed a negative mean asynchrony but there were no significant group differences in the mean asynchrony or mean IRIs in baseline conditions. The DJ group produced more regular timing intervals overall in baseline conditions, and a similar, non-significant trend for the asynchrony standard deviations across the three groups was observed. In the distractor conditions, the distractor metronome contributed significantly to the variability of the tapping asynchronies, but the DJ group achieved a lower asynchrony variability overall when compared to the NM group, and were unaffected by large positive distractor phase-offsets that caused a large increase in variability in the other two groups.

Musicians' superiority over non-musicians at minimising asynchrony during general tapping tasks is well established in the literature (Repp 1999, 2003b; Krause et al. 2010a, 2010b; Fuji et al. 2011) however these results could not reliably affirm claims that classically trained musicians showed a smaller negative mean asynchrony (NMA) in relation to other populations (Aschersleben 2002). For example, in Aschersleben's (2002) study, the NMAs of musicians were as low as $-14 \mathrm{~ms}$ in relation to non-musician populations, whose taps anticipated the metronome by up to $50 \mathrm{~ms}$, but the findings of the current research did not reveal significant differences in NMA between groups, and most participants fell consistently between -30 and $-45 \mathrm{~ms}$. In the Repp (2003a) study on which the distractor condition used in this study was based, Repp found that phase differences between a target and distractor metronome exerted a strong effect on synchronization accuracy and the variability of taps, a phenomenon which Repp called "phase attraction". Supporting Repp's findings, the effects of phase attraction were also evident in all populations in this study, however, a key finding is that DJ participants were least variable of all the groups when faced with large phase-distractor offsets that increased variability in the other two groups, an advantage that may be due to regular beatmatching of two unsynchronised tracks during the mixing process. However, it should be noted that contemporary DJ equipment provides the possibility of automatically beatmatching tracks, and it is therefore debatable as to how long beatmatching will remain an element of DJ performance as certain DJing genres become increasingly more automated (e.g. House/Techno). It is suggested that the practice of beatmatching should still be a requirement for any formal DJing instruction that aims to develop SMS abilities.

Further research would build on these preliminary findings; results illustrate that the need for a large-scale study that is broader in scope. Research could also be directed towards establishing a more comprehensive understanding of the range of SMS skills developed through DJing. Suggestions include an adaptive timing study in which phase perturbations/ shifts occurring in the target sequence could be used to investigate phase correction responses (measured through phase shifts below point of JND - =/ $10 \%$ of the IOI) (Repp 2010) and period correction (measured through the use of phase shifts above point of JND) (Schulze et al. 2005). The degree to which formal musical training and informal musical practices enhances other cognitive faculties (e.g. executive function, working memory) should also be the focus of future research, though it should be noted that musical training should not have to justify itself according to its extra-musical outcomes, in agreement with Rauscher (2009). 
The results of the qualitative study highlighted that participants in all three groups felt that DJs learn valuable musical skills that are not specific to DJing but to music education generally, including rhythm perception, harmonic perception, instrumental skills, knowledge of musical structure, performance skills and stylistic knowledge. A majority agreed that DJing had equal relevance with other musical forms (e.g. classical music) and that DJing had a high degree of contemporary relevance, especially in popular culture. On the whole, non-musicians were the most sceptical of the value of DJing in society and education, and DJs that were also DJing tutors had the most positive attitudes towards the inclusion of DJing into formal music education. An overwhelming majority of participants agreed that DJing lessons should be offered alongside traditional instrumental tuition, yet there is much progress to be made in establishing criteria for the examination of DJ performances to enable young people to pursue DJing at school. To establish the sort of instrumental training using DJing equipment that formal music education requires (Green 2002), formal links will need to be made between music education institutions and DJing academies and teachers. How DJing might be more generally incorporated into school music curriculums and examinations is deserving of future research, directed towards investigating the views of a wide sample group of formal music educators and amateur DJs, who may help us to gain insight into the way in which these musicians acquire instrumental skills and musical knowledge in much the same way as Green's (2002) investigations of popular musicians informal learning practices. Such research would provide data that could be used to articulate how an educational methodology for DJing lessons might be structured and implemented and eventually integrated into formal music education.

In the qualitative study, the views of classically trained performers and non-musicians were restricted to short responses. Future research could build a more comprehensive data set on perspectives regarding DJing in order to develop awareness of its educational potential as well as its usefulness for those with Special Education Needs. In addition, as it was difficult to reliably establish levels of formal and informal training through self-report and certain markers of achievement (e.g. formal examinations or measures of professional experience), longitudinal studies might be conducted in order to quantify the relationship between amount of time spent practicing and musical outcomes while devising a way to measure the degree to which formally trained musicians adopt informal training strategies to learn a number of other instruments which they are familiar with but not expert in (as well as applying informally learned techniques to their formally learned art).

\section{CONCLUSION}

The aim of this research was to establish the skills learned through DJing and to map perspectives on the potential value of DJing for music education from a variety of people involved in music performance and education. In line with Green's (2002) view that informal learning practices and formal music education are not mutually exclusive but draw on one another, the findings substantiate the view that contemporary music education could be strengthened by integrating practices that have grown organically in informal contexts and 
have subsequently developed into socially significant musical practices. DJing, as a culturally relevant informal musical practice, should be placed firmly on the research agenda in the fields of music education and music psychology.

\section{REFERENCES}

Aschersleben, Gisa. 1994. Afferente Informationen und die Synchronisation von Ereignissen. Lang: Frankfurt.

Aschersleben, Gisa. 2002. "Temporal Control of Movements in Sensorimotor Synchronization". Brain and Cognition 48(1): 66-79. <http://dx.doi.org/10.1006/ brcg.2001.1304>.

Attias, Bernardo, Anna Gavanas, and Hillegonda Rietveld, eds. 2013. DJ Culture, In the Mix: Power, Technology, and Social Change in Electronic Dance Music. London: Bloomsbury.

Bakker, Hans J. I. and Baker, Theo T. R. A. 2006. "The Club DJ: A Semiotic and Interactionist Analysis”. Symbolic Interaction 29(1): 71-82. < http://dx.doi.org/10.1525/si.2006.29.1.71>.

Bell, Paul. 2009. "Interrogating the Live: A DJ Perspective." Doctoral Thesis, Newcastle University, UK.

Bradby, Barbara. 1993. "Sampling Sexuality: Gender, Technology and the Body in Dance Music". Popular Music 12(02): 155-76. <http://dx.doi.org/10.1017/s0261143000005535>.

Brewster, Bill, and Frank Broughton. 2012. The Record Players: The Story of Dance Music Told by History's Greatest DJs. United Kingdom: Virgin Books.

Broughton, Frank, and Bill Brewster. 2002. How to DJ (properly): The Art and Science of Mixing. London: Bantam Press.

Butler, Mark J. 2006. Unlocking the Groove: Rhythm, Meter, and Musical Design in Electronic Dance Music. Bloomington: Indiana University Press.

Butler, Blake E. and Laurel J. Trainor. 2015. "The Musician Redefined: A Behavioral Assessment of Rhythm Perception in Professional Club DJs". Timing \& Time Perception 3(1-2): 116-32. $<$ http://dx.doi.org/10.1163/22134468-03002041>.

Cain, Tim. 2013. “Passing It On: Beyond Formal or Informal Pedagogies”. Music Education Research 15(1): 74-91. <http://dx.doi.org/10.1080/14613808.2012.752803>.

Chang, Jeff. 2005. Can't Stop Won't Stop: A History of the Hip-Hop Generation. London: Edbury Press.

Chapman, Dale E. 2012. “Turntablism”. In Grove Music Online and Oxford Music Online. Oxford University Press. $<$ http://0-www.oxfordmusiconline.com.wam.leeds.ac.uk/subscriber/article/ grove/music/A2225770>. (Accessed on 31 December 2014).

Crow, Bill. 2006. "Musical Creativity and the New Technology". Music Education Research 8(1): 121-30. <http://dx.doi.org/10.1080/14613800600581659>.

de Lissandri, Dan interview (at the DJ School, Leeds) on 11 April 2014.

Elliott, Mark T., Andrew E. Welchman and Alan M. Wing. 2009. "MatTAP: A MATLAB Toolbox for the Control and Analysis of Movement Synchronisation Experiments". Journal of Neuroscience Methods 177(1): 250-7. <http://dx.doi.org/10.1016/j.jneumeth.2008.10.002>. 
Elliott, Mark T., Alan M. Wing, and Andrew E. Welchman. 2011. "The Effect of Ageing on Multisensory Integration for the Control of Movement Timing". Experimental Brain Research 213(2-3): 291-98. <http://dx.doi.org/10.1007/s00221-011-2740-x>.

Engbert, Ralf, Ralf T. Krampe, Jürgen Kurths and Reinhold Kliegl. 2002. "Synchronizing Movements with the Metronome: Nonlinear Error Correction and Unstable Periodic Orbits". Brain and Cognition 48(1): 107-16. <http://dx.doi.org/10.1006/brcg.2001.1307>.

Fujii, Shinya, Masaya Hirashima, Kazutoshi Kudo, Tatsuyuki Ohtsuki, Yoshihiko Nakamura, and Shingo Oda. 2011. "Synchronization Error of Drum Kit Playing with a Metronome at Different Tempi by Professional Drummers”. Music Perception: An Interdisciplinary Journal 28(5): 491-503. <http://dx.doi.org/10.1525/mp.2011.28.5.491>.

Greasley, Alinka E. and Helen M. Prior. 2013. "Mixtapes and Turntablism: DJs' Perspectives on Musical Shape”. Empirical Musicology Review 8(1): 23-43. < http://dx.doi.org/10.18061/emr. v8i1.3921>.

Green, Lucy. 2002. How Popular Musicians Learn. Burlington: Ashgate.

-_- 2009. Music, Informal Learning and the School: A New Classroom Pedagogy. Burlington: Ashgate Publishing.

Hallam, Susan., and Lynne Rogers. 2010. "Creativity". In Music Education in the 21st Century in the United Kingdom: Achievements, Analysis and Aspirations, ed. Susan Hallam and Andrea Creech, 105-22. London: Institute of Education, University of London.

Hansen, Kjetil F. 2010. “The Acoustics and Performance of DJ Scratching: Analysis and Modelling”. Doctoral Thesis, KTH Royal Institute of Technology. < http://www.speech.kth.

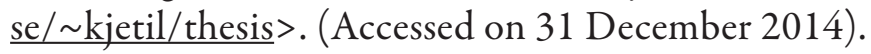

Himonides, Evangelos and Ross Purves. 2010. “The Role of Technology”. In Music Education in the 21st Century in the United Kingdom: Achievements, Analysis and Aspirations, ed. Susan Hallam and Andrea Creech, 123-40. London: Institute of Education, University of London.

Jabusch, Hans-Christian. 2006. "Movement Analysis in Pianists". In Music, Motor Control and the Brain, ed. Eckhart Altenmüller, Mario Wiesendanger and Jurg Kesselring, 91-108. Oxford: Oxford University Press.

Jäncke, Lutz. 2006. "From Cognition to Action”. In Music, Motor Control and the Brain, ed. Eckhart Altenmüller, Mario Wiesendanger and Jurg Kesselring, 26-37. Oxford: Oxford University Press.

Katz, Mark N. 2012. Groove Music: The Art and Culture of the Hip-Hop DJ. New York: Oxford University Press.

Kistner, Gavin. 2006. "Hip-Hop Sampling and Twentieth Century African-American Music: An Analysis of Nas' 'Get Down (2003)'”. Doctoral Dissertation, Université Laval.

Konvalinka, Ivana, Peter Vuust, Andreas Roepstorff, and Chris D. Frith. 2010. "Follow You, Follow Me: Continuous Mutual Prediction and Adaptation in Joint Tapping”. Quarterly Journal of Experimental Psychology (2010) 63(11): 2220-30. < http://dx.doi.org/10.1080/174 70218.2010.497843>.

Krampe, Ralf T, Ralf Engbert, and Reinhold Kliegl. 2001. "Age-specific Problems in Rhythmic Timing”. Psychology and Aging 16(1): 12-30. < http://dx.doi. org/10.1037/0882-7974.16.1.12>. 
Krause, Vanessa, Alfons Schnitzler, and Bettina Pollok. 2010a. "Functional Network Interactions During Sensorimotor Synchronization in Musicians and Non-Musicians". Neuroimage 52(1): 245-51. <http://dx.doi.org/10.1016/j.neuroimage.2010.03.081 >.

- - 2010b. "Perception in Action: The Impact of Sensory Information on Sensorimotor Synchronization in Musicians and Non-Musicians". Acta Psychologica 133(1): 28-37. <http:// dx.doi.org/10.1016/j.actpsy.2009.08.003>.

Luck, Geoff, and Petri Toiviainen. 2006. “Ensemble Musicians' Synchronization with Conductors' Gestures: An Automated Feature-Extraction Analysis". Music Perception 24(2): 189-200. <http://dx.doi.org/10.1525/mp.2006.24.2.189>.

Madison, Guy. 2000. "On the Nature of Variability in Isochronous Serial Interval Production”. In Rhythm Perception and Production, ed. Peter Desain and Luke Windsor, 95-113. Lisse: Swets and Zeitlinger.

Marsh, Charity. 2006. "In \& Out of the Classroom: Reflections on Identity, Technology, and the Radio Project". Intersections: Canadian Journal of Music Intersections:/Revue Canadienne de Musique 26(2): 81-96. <http://dx.doi.org/10.7202/1013228ar>.

_- _ 2012. "Bits and Pieces of Truth: Storytelling, Identity, and Hip Hop in Saskatchewan”. Perspectives on Contemporary Aboriginal Music in Canada, 346-71.

McQueen, Hilary, and Susan Hallam. 2010. "Music in the Secondary School”. In Music Education in the 21st Century in the United Kingdom: Achievements, Analysis and Aspirations, ed. Susan Hallam and Andrea Creech, 228-44. London: Institute of Education, University of London.

Montano, Ed. 2010. “How Do You Know He's Not Playing Pac-Man While He’s Supposed to be DJing? Technology, Formats and the Digital Future of DJ Culture". Popular Music 29(3): 397-416. < http://dx.doi.org/10.1017/s0261143010000449>.

- - - 2013. "DJ Culture and the Commercial Club Scene in Sydney". In DJ Culture, In the Mix: Power, Technology, and Social Change in Electronic Dance Music, ed. Attias, Bernardo, Anna Gavanas, and Hillegonda Rietveld, 173-94. London: Bloomsbury.

Musical Futures, 2014. < https://www.musicalfutures.org>. (Accessed on 12 December 2014).

Nowicki, Lena, Wolfgang Prinz, Marc Grosjean, Bruno H. Repp, and Peter E. Keller. 2013.

"Mutual Adaptive Timing in Interpersonal Action Coordination".

Psychomusicology: Music, Mind, and Brain 23(1): 6-20. <http://dx.doi.org/10.1037/a0032039>.

Pasagiannis, John. P. 2007. "Hip Hop Music Treatment With At-Risk Adolescent Populations”. PhD Dissertation. Gordon F. Derner Institute of Advanced Psychological Studies, Adelphi University, NY.

Poschardt, Ulf. 2002. DJ Culture. Paris: Editions Kargo.

Price, David, and Abilgail D’Amore. 2007. From Vision to Practise: Musical Futures: A Summary of Key Findings". London: The Paul Hamlyn Foundation.

Rauscher, Frances H. 2009. "The Impact of Music Instruction on Other Skills”. In The Oxford Handbook of Music Psychology, ed. Susan Hallam and Andrea Creech, 244-52. Oxford: Oxford University Press.

Reiss, Jim. Interview (at the DJ School, Leeds) on 1 April 2014.

Repp, Bruno. H. 1999. "Control of Expressive and Metronomic Timing in Pianists”. Journal of Motor Behavior 31(2): 145-64. <http://dx.doi.org/10.1080/00222899909600985>. 
- - -. 2002. "Automaticity and Voluntary Control of Phase Correction Following Event Onset Shifts in Sensorimotor Synchronization”. Journal of Experimental Psychology: Human Perception and Performance 28(2): 410-30. < http://dx.doi. org/10.1037//0096-1523.28.2.410>.

- - - 2003a. "Phase Attraction in Sensorimotor Synchronization with Auditory Sequences: Effects of Single and Periodic Distractors on Synchronization Accuracy". Journal of Experimental Psychology: Human Perception and Performance 29(2): 290-309. < http:// dx.doi.org/10.1037/0096-1523.29.2.290>.

_- _. 2003b. "Rate Limits in Sensorimotor Synchronization with Auditory and Visual Sequences: The Synchronization Threshold and the Benefits and Costs of Interval Subdivision". Journal of Motor Behavior 35(4): 355-70. <http://dx.doi. org/10.1080/00222890309603156>.

- - 2005. "Sensorimotor Synchronization: A Review of the Tapping Literature". Psychonomic Bulletin \& Review 12(6): 969-92. <http://dx.doi.org/10.3758/bf03206433>.

_-_.. 2010. "Sensorimotor Synchronization and Perception of Timing: Effects of Music Training and Task Experience”. Human Movement Science 29(2): 200-13. < $\underline{\text { http://dx.doi. }}$ org/10.1016/j.humov.2009.08.002>.

Repp, Bruno H., and Rebecca Doggett. 2007. “Tapping to a Very Slow Beat: A Comparison of Musicians and Nonmusicians”. Music Perception: An Interdisciplinary Journal 24(4): 367-76. <http://dx.doi.org/10.1525/mp.2007.24.4.367>.

Repp, Bruno H., and Gordon P. Moseley. 2012. "Anticipatory Phase Correction in Sensorimotor Synchronization”. Human Movement Science 31(5): 1118-36. < http://dx.doi.org/10.1016/j. humov.2011.11.001>.

Repp, Bruno H., and Yi-Huang Su. 2013. "Sensorimotor Synchronization: A Review of Recent Research (2006-2012)”. Psychonomic Bulletin \& Review 20(3): 403-52. < http://dx.doi. org/10.3758/s13423-012-0371-2>.

Schulze, Hans-Henning, Andreas Cordes, and Dirk Vorberg. 2005. "Keeping Synchrony While Tempo Changes: Accelerando and Ritardando". Music Perception 22(3): 461-77. < http:// dx.doi.org/10.1525/mp.2005.22.3.461>.

Shiu, Anthony Sze-Fai. 2007. "Styl (us): Asian North America, Turntablism, Relation”.CR: The New Centennial Review 7(1): 81-106. < http://dx.doi.org/10.1353/ncr.2007.0029>.

Smith, Sophy. 2000. “Compositional Strategies of the Hip-Hop Turntablist”. Organised Sound 5(2): 75-79. < http://dx.doi.org/10.1017/s135577180000203x >.

- - - 2007. "The Process of 'Collective Creation' in the Composition of UK Hip-Hop Turntable Team Routines”. Organised Sound 12(01): 79-87. < http://dx.doi.org/10.1017/ $\underline{\text { s1355771807001677>. }}$

- - . 2013. Hip-Hop, Turntablism, Creativity and Collaboration. Burlington: Ashgate.

Snell, Karen. 2011. "Turntablism: A Vehicle for Connecting Community and School Music Making and Learning". In Pop-Culture Pedagogy in the Music Classroom: Teaching Tools from American Idol to YouTube, ed. Nicole Biamonte, 173-84. Plymouth: Scarecrow Press.

Steventon, John. 2010. DJing for Dummies (2nd Ed.). Chichester: John Wiley \& Sons. Thomas, Douglas, and John Seely Brown. 2011. A New Culture of Learning: Cultivating the Imagination for a World of Constant Change. Lexington: Create Space Lexington. 
Toiviainen, Petri, and Joel S. Snyder. 2003. “Tapping to Bach: Resonance-based Modeling of Pulse”. Music Perception: An Interdisciplinary Journal 21(1): 43-80. <http://dx.doi. org/10.1525/mp.2003.21.1.43>.

Van Dyck, Edith, Dirk Moelants, Michiel Demey, Alexander Deweppe, Pieter Coussement, and Marc Leman. 2013. "The Impact of the Bass Drum on Human Dance Movement”. Music Perception: An Interdisciplinary Journal 30(4): 349-59. < http://dx.doi.org/10.1525/ mp.2013.30.4.349>.

Vandemast-Bell, Paul. 2013. "Rethinking Live Electronic Music: A DJ Perspective". Contemporary Music Review 32(2-3): 239-48. <http://dx.doi.org/10.1080/07494467.2013.775817>.

Vorobyev, Yakov, and Eric Coomes. 2012. Beyond Beatmatching: Take Your DJ Career to the Next Level. E-book: Mixed in Key Publications.

Webber, Stephen. 2007. DJ Skills: The Essential Guide to Mixing and Scratching. Burlington, MA: Focal Press.

Wheeler, Steve, S. J. Waite, and Carolyn Bromfield. 2002. "Promoting Creative Thinking Through the Use of ICT". Journal of Computer Assisted Learning 18(3): 367-78. < $\underline{\text { http://dx.doi.org/10 }}$ $.1046 / \mathrm{j} .0266-4909.2002 .00247 . \mathrm{x}>$.

Wing, Alan M., Satoshi Endo, Adrian Bradbury, and Dirk Vorberg. 2014. "Optimal Feedback Correction in String Quartet Synchronization”. Journal of the Royal Society Interface 11(93): 1125. <http://dx.doi.org/10.1098/rsif.2013.1125>. 